\title{
Alternative Ii-independent antigen-processing pathway in leukemic blasts involves TAP-dependent peptide loading of HLA class II complexes
}

\author{
Marvin M. van Luijn • Martine E. D. Chamuleau • Maaike E. Ressing • Emmanuel J. Wiertz • \\ Suzanne Ostrand-Rosenberg • Yuri Souwer • Adri Zevenbergen • Gert J. Ossenkoppele • \\ Arjan A. van de Loosdrecht • S. Marieke van Ham
}

Received: 26 March 2010/Accepted: 14 August 2010/Published online: 5 September 2010

(C) The Author(s) 2010. This article is published with open access at Springerlink.com

\begin{abstract}
During HLA class II synthesis in antigen-presenting cells, the invariant chain (Ii) not only stabilizes HLA class II complexes in the endoplasmic reticulum, but also mediates their transport to specialized lysosomal antigen-loading compartments termed MIICs. This study explores an alternative HLA class II presentation pathway in leukemic blasts that involves proteasome and transporter associated with antigen processing (TAP)-dependent peptide loading. Although HLA-DR did associate with Ii, Ii silencing in the human class II-associated invariant chain peptide (CLIP)-negative KG-1 myeloid leukemic cell line did not affect total and plasma membrane expression levels
\end{abstract}

Electronic supplementary material The online version of this article (doi:10.1007/s00262-010-0908-z) contains supplementary material, which is available to authorized users.

M. M. van Luijn $(\varangle) \cdot$ M. E. D. Chamuleau - A. Zevenbergen ·

G. J. Ossenkoppele · A. A. van de Loosdrecht

Department of Hematology, Cancer Center Amsterdam,

VU Institute for Cancer and Immunology,

VU University Medical Center, De Boelelaan 1117,

1081 HV Amsterdam, The Netherlands

e-mail: m.luijn@vumc.nl

M. M. van Luijn - Y. Souwer · S. M. van Ham

Department of Immunopathology, Sanquin Research

and Landsteiner Laboratory, Academic Medical Center,

University of Amsterdam, Plesmanlaan 125,

1066 CX Amsterdam, The Netherlands

M. E. Ressing · E. J. Wiertz

Department of Medical Microbiology, University Medical Center Utrecht, Utrecht and Leiden University Medical Center, Leiden, The Netherlands

S. Ostrand-Rosenberg

Department of Biological Sciences, University of Maryland,

Baltimore County, Baltimore, MD, USA of HLA-DR, as determined by western blotting and flow cytometry. Since HLA-DR expression does require peptide binding, we examined the role of endogenous antigenprocessing machinery in HLA-DR presentation by CLIP ${ }^{-}$ leukemic blasts. The suppression of proteasome and TAP function using various inhibitors resulted in decreased HLA-DR levels in both CLIP $^{-}$KG-1 and ME-1 blasts. Simultaneous inhibition of TAP and Ii completely downmodulated the expression of HLA-DR, demonstrating that together these molecules form the key mediators of HLA class II antigen presentation in leukemic blasts. By the use of a proteasome- and TAP-dependent pathway for HLA class II antigen presentation, CLIP $^{-}$leukemic blasts might be able to present a broad range of endogenous leukemiaassociated peptides via HLA class II to activate leukemiaspecific $\mathrm{CD} 4^{+} \mathrm{T}$ cells.

Keywords MHC class II - Tumor cells · Antigen presentation $\cdot$ Leukemia $\cdot$ Immune surveillance

\section{Introduction}

Professional antigen-presenting cells (APC), such as dendritic cells (DCs) and macrophages, can activate $\mathrm{T}$ cells by efficiently processing antigens from both endogenous and exogenous sources and presenting them at the plasma membrane.

HLA class I antigen-processing machinery is essential for the generation of endogenous peptides and loading onto HLA class I molecules in the endoplasmic reticulum (ER). One of its major components is the proteasome, a multicatalytic proteinase complex that specifically cleaves ubiquitin-conjugated cytoplasmic proteins into small peptides [1]. Another key player, the transporter associated 
with antigen processing (TAP), mediates translocation of these peptides into the ER [2], where further trimming to nonameric peptides may be necessary for HLA class I binding [3]. Upon complete folding in the ER lumen, the class I/peptide complex is exported via the default secretory pathway to the plasma membrane.

Exogenously derived peptides are generated in the endosomal/lysosomal pathway and loaded onto HLA class II molecules in specialized lysosomal antigen-loading compartments, termed MIICs (or MHC class II-containing compartments) [4, 5]. Newly synthesized HLA class II $\alpha \beta$ heterodimers associate with the invariant chain (Ii or CD74) in the ER lumen, which not only ensures stabilization and proper folding, but also transport to the MIICs $[6,7]$. During transport to the MIICs, Ii is proteolytically degraded, leaving a small fragment bound to the peptidebinding groove [8]. In the MIICs, this class II-associated invariant chain peptide (CLIP) is exchanged for an exogenous peptide by the peptide editor HLA-DM (DM) [9], and the class II/peptide complex is transported to the plasma membrane.

As extensively described in myeloid DCs, exogenous peptides are able to interact with HLA class I molecules via different processing pathways, a mechanism known as cross-presentation $[10,11]$. Endogenous peptides, on the other hand, can be loaded onto HLA class II molecules as well, although it remains controversial which processing pathways underlie this type of peptide loading. In many professional APCs, endogenous antigens are transferred to the endosomal/lysosomal pathway for loading onto HLA class II molecules [12]. Autophagy has been reported to play a role in this process by delivering cytoplasmic material for degradation in the lysosomes [13-15], but also the proteasome could be involved by degrading cytoplasmic antigens before entering the endosomal/lysosmal pathway $[16,17]$.

Although HLA class II transport into this pathway is classically known to be dependent of Ii, the absence of Ii in tumor APCs was shown to clearly increase HLA class IIrestricted presentation of endogenous peptides and their capacity to activate tumor-specific $\mathrm{CD}^{+} \mathrm{T}$ cells $[18,19]$. Taking into account that the association of Ii with HLA class II interferes with endogenous peptide binding in the ER [20, 21], this suggests that in certain tumor APCs, loading of HLA class II molecules with endogenous peptides occurs via HLA class I antigen-processing machinery instead of the endosomal/lysosomal pathway. It has recently been shown that both the proteasome and TAP were involved in the processing of HLA class II-restricted viral epitopes [22], but less is known about this function of HLA class I antigen-processing machinery in HLA class $\mathrm{II}^{+}$tumor APCs. A better understanding of endogenous antigen processing for HLA class II-restricted presentation in HLA class $\mathrm{II}^{+}$tumor cells could be important for developing approaches to optimize presentation of, yet unidentified, tumor-associated antigens (TAAs), thereby circumventing tumor immune escape.

We previously demonstrated that a low amount of CLIP on HLA class $\mathrm{II}^{+}$leukemic blasts predicts an improved clinical outcome for patients with acute myeloid leukemia (AML) and enhances recognition by allogeneic $\mathrm{CD}^{+}{ }^{+} \mathrm{T}$ cells [23]. In the current study, we show that an Ii-independent, but proteasome- and TAP-dependent HLA class II presentation pathway is active in a human CLIP ${ }^{-}$ myeloid leukemic cell line, which indicates that HLA class I antigen-processing machinery mediates HLA class II-restricted presentation of endogenous peptides in these tumor cells.

\section{Materials and methods}

Cell lines and culturing

The human myeloid leukemic cell lines KG-1 and Kasumi-1 were obtained from the American Type Culture Collection (ATTC) and frozen in aliquots at low cell passage. Culturing of the KG-1 cell line was performed at $3 \times 10^{5}$ cells $/ \mathrm{ml}$ in IMDM (Gibco) supplemented with $20 \%$ FBS, 1\% L-glutamine (Gibco), 25 mM Hepes (Sigma-Aldrich, St Louis, MO, USA) and $50 \mu \mathrm{M}$ 2-ME (Gibco). The Kasumi-1 cell line was maintained in RPMI 1640 medium (Gibco, Paisley, UK) supplemented with $1 \%$ L-glutamine and $15 \%$ heatinactivated FBS (Greiner, Alphen a/d Rijn, The Netherlands) with a cell density of $5 \times 10^{5}$ cells $/ \mathrm{ml}$. Cells were incubated at $37^{\circ} \mathrm{C}$ in a humidified atmosphere containing $5 \% \mathrm{CO}_{2}$ and the culture medium was refreshed every 3 days.

\section{Antibodies and immunofluorescence staining}

The following mouse monoclonal antibodies (MoAbs) were used: PE-labeled anti-HLA-ABC (W6/32 clone; Dako, Glostrup, Denmark) and $\mathrm{IgG}_{2 \mathrm{a}}$ isotype control (BD, San Jose, CA, USA); APC-labeled anti-HLA-DR (clone L243) and $\mathrm{IgG}_{2 \mathrm{a}}$ isotype control (BD); and 7-amino-actinomycin D (7AAD; Via-Probe, BD). The PIN1.1 antibody (anti-Ii) was kindly provided by Peter Cresswell (Howard Hughes Medical Institute, Yale University School of Medicine, New Haven, CT, USA) [24].

For immunofluorescence stainings, $1 \times 10^{5}$ cells were pre-incubated with $10 \%$ human gamma-globulin $(60 \mathrm{mg} / \mathrm{ml}$; Sanquin) for $10 \mathrm{~min}$. Mouse MoAbs were added during $15 \mathrm{~min}$ for extracellular and $30 \mathrm{~min}$ for intracellular staining. To analyze total protein expression, cells were fixed with PBS 1\% paraformaldehyde, permeabilized using PBS $0.1 \%$ saponin (Sigma-Aldrich) and stained with the 
MoAb of interest. We examined the total amount of Ii by staining with PIN1.1 followed by a second incubation step of 20 min with rabbit anti-mouse (RAM) IgG conjugated to PE (Dako). Stained cells were incubated at room temperature (RT), washed twice with PBS containing $0.1 \%$ HSA and $0.05 \%$ sodium azide, and measured on a FACSAria flow cytometer (BD). The same apparatus was used for flow cytometric sorting of cells. Sorted cells were washed three times with PBS before subsequent analysis or culture in medium. We performed flow cytometric analyses by using CellQuest software (BD) and determined extracellular expression levels on viable $\left(7 \mathrm{AAD}^{-}\right)$cells. Mean fluorescence intensity (MFI) was defined as the median value of all gated cells relative to that of isotype control staining.

DNA constructs and retroviral vectors

Epstein-Barr virus (EBV)-encoded BNLF2a and bovine herpes virus 1 (BHV-1)-encoded UL49.5 were amplified from viral DNA with specifically selected primers and PCR products were cloned into pLZRS-IRES-EGFP, as previously reported $[25,26]$. Information about this vector can be obtained at http://www.stanford.edu/group/nolan/ retroviral_systems/retsys.html. Ii silencing was performed using a pSIREN-RetroQ retroviral vector (Clontech) that contains a specifically prepared Ii-siRNA insert (sequence 53) with a puromycin resistance gene [27].

Retroviral production, transductions and transfections

BNLF2a and UL49.5 retroviruses were produced using the Phoenix amphotropic packaging system, as described at http://www.stanford.edu/group/nolan/protocols/pro_helper_ dep.html. For the production of UL49.5 retroviruses, we used a pLZRS vector that encodes a mutant form of UL49.5, containing two alanines instead of lysines within the cytoplasmic tail. This construct did not alter UL49.5mediated degradation and inhibition of the TAP complex [28]. Retroviral transductions and transfections were performed according to standard protocols [23]. The possibility of off-target effects during these procedures were excluded by using an irrelevant GFP-encoding retrovirus (supplementary material, Fig. S2) and irrelevant siRNAs [27].

For transfection experiments, $2 \mu \mathrm{g}$ of pSIREN-RetroQIi-siRNA53 DNA was delivered into $2 \times 10^{6}$ cells using the Amaxa nucleofection technology (Lonza, Basel, Switzerland). The optimized protocol for the KG-1 cell line is available at http://www.lonzabio.com. Before treated cells were resuspended in culture medium again, we first recovered them in IMDM (Gibco) without the addition of supplements for $30 \mathrm{~min}$ at $37^{\circ} \mathrm{C}$. Two days after transfection, we added $1.0 \mu \mathrm{g} / \mathrm{ml}$ puromycin to select IisiRNA53-transfected cells.

\section{Co-immunoprecipitations and western blotting}

To immunoprecipitate $\mathrm{Ii}$ proteins, $10 \times 10^{6}$ myeloid leukemic blasts were washed three times with sterile PBS ( $\mathrm{pH}$ 7.4). Cell lysates were prepared by snap freezing the samples in liquid nitrogen and incubating them upon thawing for $45 \mathrm{~min}$ in $250 \mu \mathrm{l}$ ice-cold lysis buffer, which consisted of sterile PBS supplemented with $1 \%$ Ipegal and $15 \%$ protease inhibitor cocktail (Complete; 1 tablet $/ 7.5 \mathrm{ml}$ $\mathrm{H}_{2} \mathrm{O}$; Boehringer Mannheim Biochemica, Mannheim, Germany). After removal of unlysed cell remnants and nuclei by centrifugation in an Eppendorf micro-centrifuge (5 min, 10,000 rpm), the protein content of the supernatant was determined by the Bio-Rad protein assay (Biorad Laboratories, Hercules, CA, USA). Then, $0.5 \mathrm{mg}$ total cell lysates were diluted in $0.5 \mathrm{ml}$ ice-cold lysis buffer and precleared with $1.0 \mu \mathrm{g}$ of mouse $\mathrm{IgG}_{1} \mathrm{MoAb}$ together with $20 \mu$ Protein G PLUS-Agarose reagent (Santa Cruz Biotechnology, Santa Cruz, CA, USA) for $30 \mathrm{~min}$. Ii was immunoprecipitated using the PIN1.1 antibody and Protein G PLUS-Agarose reagent $(20 \mu \mathrm{l})$ in an overnight incubation. Ii immunoprecipitates were collected by centrifugation and washed four times with PBS before resuspending in reducing electrophoresis sample buffer.

For western blotting, samples were electrophoresed on $12.5 \%$ polyacrylamide gels that contained SDS and transferred onto methanol-activated PVDF membranes. Different protein amounts from total cell lysates were loaded on gel: $10 \mu \mathrm{g}$ for HLA-DR $\alpha, 20 \mu \mathrm{g}$ for Ii and $70 \mu \mathrm{g}$ for TAP1. Mouse anti-DR $\alpha$ (clone 1B5 [29], 1:10,000), anti-Ii (clone PIN1.1 [24], 1:1,000) and anti-TAP1 (clone 148.3 [30], 1:100) were used as primary MoAbs. To lower the staining of interfering immunoprecipitated heavy and light chains, mouse Trueblot $^{\mathrm{TM}}$ ULTRA reagent (eBioscience, San Diego, CA, USA) was added. Protein complexes were visualized by Amersham ECL Western Blotting Detection Reagents (GE Healthcare, Buckinghamshire, UK).

Proteasome inhibition assays

MG-132 and bortezomib (PS-341, Velcade) were kind gifts from Dr. Gerrit Jansen (VU University Medical Center, Amsterdam, The Netherlands). Each of these inhibitors was added to $5 \times 10^{5}$ cells $/ \mathrm{ml}$ culture medium per well in increasing concentrations using six-well plates and cells were incubated overnight at $37^{\circ} \mathrm{C}$ in a humidified atmosphere containing $5 \% \mathrm{CO}_{2}$. Following incubation, cells were washed twice with PBS containing $0.1 \%$ HSA and $0.05 \%$ sodium azide and centrifuged for immunofluoresence staining. 


\section{Results}

HLA-DR expression and transport to the plasma membrane in $\mathrm{CLIP}^{-} \mathrm{KG}-1$ blasts can occur in the absence of the invariant chain

To study the Ii-dependency of HLA-DR expression by CLIP $^{-}$leukemic blasts, we silenced Ii expression in the KG-1 myeloid leukemic cell line, which was found to lack CLIP at the plasma membrane [23].

Western blot analysis demonstrated that Ii expression was strongly inhibited in Ii-siRNA-treated KG-1 (KG-1/ Ii-siRNA) blasts (Fig. 1a). Interestingly, the total amount of HLA-DR in KG-1/Ii-siRNA blasts was similar to that in wild-type blasts. These results were confirmed by flow cytometry, which showed that Ii was down-regulated for $>98 \%$ and, despite a very small population of HLA$\mathrm{DR}^{-} \mathrm{Ii}^{-}$blasts, total HLA-DR expression remained unaffected in the vast majority of permeabilized, Ii-negative KG-1/Ii-siRNA blasts (Fig. 1b, left panels).

Since the formation of HLA-DR molecules was not impaired in KG-1/Ii-siRNA blasts, we next questioned whether their transport to the plasma membrane was disturbed by FACS analysis of HLA-DR expression on intact cells (Fig. 1b, right panel). Again, no differences in expression were encountered, implicating that the absence of Ii does not interrupt HLA-DR transport to the plasma membrane.

To assess if this Ii-independent HLA-DR expression resulted from an inability of Ii to interact with HLA-DR molecules, we immunoprecipitated Ii from KG-1 blasts and determined its association with HLA-DR by western blotting. As a positive control, the CLIP $^{+}$Kasumi-1 myeloid leukemic cell line was used, in which HLA-DR expression did depend on the function of Ii [23]. Immunoprecipitation of Ii from these cell lines revealed a similar association with HLA-DR (Fig. 1c). Thus, the observed Ii-independent HLA-DR expression in KG-1 blasts is not a reflection of inefficient binding of Ii to HLA-DR.

These findings show that in CLIP $^{-}$KG-1 blasts, HLADR processing and transport to the plasma membrane can occur independently of Ii expression, indicating the involvement of other proteins in $\mathrm{CLIP}^{-}$leukemic blasts that stabilize HLA class II molecules.

HLA-DR expression and transport to the plasma membrane in CLIP $^{-}$KG-1 blasts is dependent on the function of the proteasome

Newly formed HLA-DR molecules are typically stabilized by Ii (and later by CLIP) prior to peptide binding in the MIICs [31, 32]. This raises the question of which alternative mechanism stabilizes these molecules in cells that do
Fig. 1 Invariant chain involvement in HLA-DR expression by KG-1 blasts. a Protein extracts from Ii-siRNA-transduced KG-1 (KG-1/ Ii-siRNA) blasts were analyzed for Ii and total HLA-DR expression by western blotting. Similar expression levels of $\beta$-tubulin verified that both lanes were equally loaded with protein. Molecular weights: Ii, $33 \mathrm{kD}$ (p33); DR $\alpha, 34 \mathrm{kD}$; $\beta$-tubulin, $55 \mathrm{kD}$. b Ii versus total HLADR expression levels for permeabilized KG-1 blasts (left panels), as well as HLA-DR expression levels at the plasma membrane of intact, 7AAD ${ }^{-}$KG-1 blasts (right panel) after treatment with Ii-siRNAs, as depicted in histograms (unfilled peaks) and dot plots. The filled histograms represent isotype control stainings of the same cells. c Coimmunoprecipitations of Ii with HLA-DR from protein extracts of KG-1 and Kasumi-1 blasts. Ii immunoprecipitates were studied for the association with HLA-DR by western blotting for $\mathrm{DR} \alpha$ under reducing conditions. Protein extracts of total lysates and $\mathrm{IgG}_{1}$ immunoprecipitates from the same blasts acted as positive and negative controls, respectively, to confirm the specificity of the DR $\alpha$ bands $(34 \mathrm{kD})$ observed in the Ii immunoprecipitate-loaded lanes

not express Ii. We studied if Ii-independent HLA-DR expression in KG-1 blasts may be accomplished by loading of cytoplasmic peptides that are processed via HLA class I antigen-processing machinery.

For classical loading of HLA class I molecules, cytoplasmic antigens need to be cleaved into small peptides by the proteasome. To investigate the role the proteasome in HLA-DR peptide loading, we blocked proteasomal activity in KG-1 blasts by two inhibitors: MG-132, which inhibits all catalytic $\beta$ subunits, and bortezomib, which specifically suppresses the $\beta 5$ and, at higher concentrations, the $\beta 1$ subunit of the $26 \mathrm{~S}$ proteasome [33].

After incubation with each inhibitor, HLA class I expression was decreased on the total KG-1 blast population, as determined by flow cytometry (Fig. 2a), indicating the effect of proteasome inhibition on endogenous peptide loading. Interestingly, HLA-DR expression was strongly down-regulated as well, in a dose-dependent manner (supplementary material, Fig. S1a). In line with its broad catalytic subunit specificity, MG-132 had a stronger effect on both HLA class I and HLA-DR expression than bortezomib (Fig. 2b). Similar effects of proteasome inhibition were found for the myeloid leukemic cell line ME-1 (supplementary material, Fig. S1b). ME-1 blasts also lack CLIP at the plasma membrane [23], indicating that our findings reflect a general mechanism for leukemic blasts that do not express CLIP.

As CLIP is abundantly expressed on Kasumi-1 blasts [23], in contrast to the KG-1 and ME-1 myeloid leukemic cell line, it is likely that cytoplasmic antigens in these blasts are not processed for presentation by HLA-DR molecules. Indeed, MG-132 and bortezomib did not reduce plasma membrane expression of HLA-DR on Kasumi-1 blasts (Fig. 2c), while HLA class I expression was affected. This demonstrates that HLA-DR expression on these blasts does not involve proteasome activity and confirms that the 
a
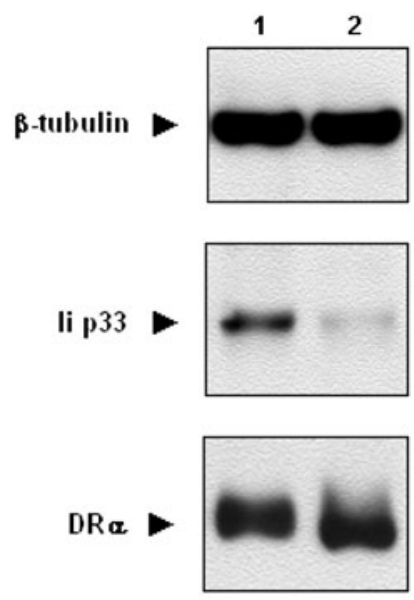

\section{$1=K G-1$}

$2=K G-1 / i-s i R N A$

b

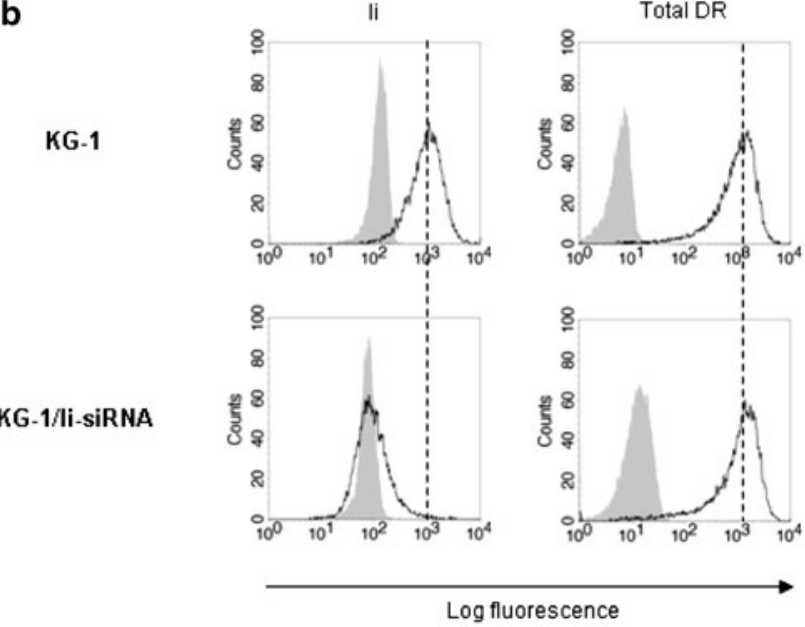

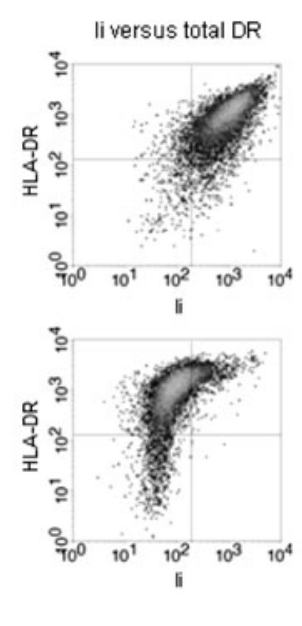

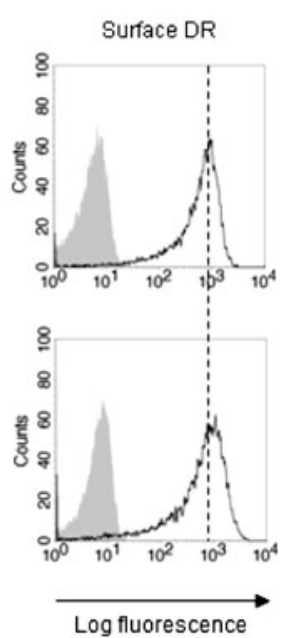

C

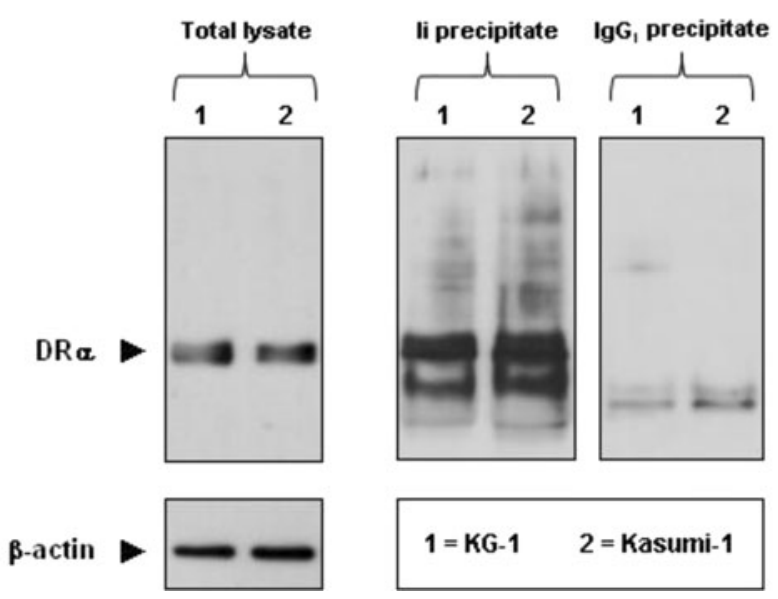


effect of both inhibitors in KG-1 and ME-1 blasts does not result from aspecific protein down-regulation.

In conclusion, these data indicate that, in contrast to CLIP $^{+}$Kasumi-1 blasts, the transport of HLA-DR molecules to the plasma membrane in at least a subpopulation of KG-1 blasts and ME-1 blasts relied on proteasomal activity, which supports the notion that Ii-independent HLA-DR expression in $\mathrm{CLIP}^{-}$leukemic blasts is related to loading with cytoplasmic peptides.

HLA-DR expression and transport to the plasma membrane in CLIP $^{-}$KG-1 blasts is partly dependent on the function of the TAP molecule

To further provide evidence that HLA class I antigenprocessing machinery is involved in cytoplasmic peptide loading of HLA-DR molecules, we suppressed TAP-mediated peptide processing in KG-1 blasts using two different viral proteins: BNLF2a, which interferes with peptide and ATP binding to TAP [25], and UL49.5, which prevents conformational changes of TAP required for peptide translocation and targets TAP for proteasomal degradation [26].

After transduction with BNLF2a/GFP- and UL49.5/ GFP-encoding retrovirus, $\mathrm{GFP}^{+} \mathrm{KG}-1$ blasts were flow cytometrically sorted and analyzed for TAP expression by western blotting (Fig. 3a). Although BNLF2a has been described to only functionally suppress the TAP complex, a lower amount of TAP1 was observed in BNLF2a-containing KG-1 (KG-1/BNLF2a) blasts, as compared to wildtype blasts. In UL49.5-containing KG-1 (KG-1/UL49.5) blasts, TAP1 expression was completely down-regulated, which agrees with the function of UL49.5 in targeting TAP for proteasomal degradation. TAP2 expression was not affected by these inhibitors, indicating that the inhibition of TAP function was mediated by down-modulation of TAP1.

To test the impact of TAP inhibition on Ii-independent HLA-DR expression, we analyzed the level of HLA-DR expression on TAP-inhibited KG-1 blasts by flow cytometry (Fig. 3b). HLA class I levels were determined as a positive control for the function of TAP in classical endogenous peptide loading. GFP ${ }^{+}$KG-1/BNLF2a and KG-1/UL49.5 blasts not only showed decreased HLA class I expression, but also a blast subpopulation with clearly reduced HLA-DR levels (total down-regulation of 21 and $36 \%$, respectively) at the plasma membrane. This effect was the strongest in $\mathrm{GFP}^{+} \mathrm{KG}-1 / \mathrm{UL} 49.5$ blasts $(53 \%$ of $\mathrm{GFP}^{+}$blasts obtained a $\mathrm{DR}^{-}$phenotype), probably as the cause of higher UL49.5 levels implicated by increased GFP expression compared to KG-1/BNLF2a blasts (Fig. 3b) and the complete degradation of TAP (Fig. 3a). In CLIP ${ }^{-}$ ME-1 blasts, TAP inhibition by using UL49.5 retrovirus resulted in down-regulation of HLA-DR on the total blast population (supplementary material, Fig. S3).
Fig. 2 KG-1 (a, b) and Kasumi-1 (c) blasts treated with various concentrations of MG-132 and bortezomib proteasome inhibitor were analyzed for plasma membrane expression of HLA class I ('ABC') and HLA-DR (unfilled histograms) together with their isotype controls (filled histograms) by flow cytometry. To define the relative MFI of HLA class I and HLA-DR expression levels, MFI values were calculated for $7 \mathrm{AAD}^{-}$blasts at each concentration as described in "Materials and methods" and related to those of untreated blasts $(n=3, \pm$ SEM $)$

To investigate whether TAP is also involved in Ii-dependent HLA-DR expression, $\mathrm{CLIP}^{+}$Kasumi-1 blasts were transduced with UL49.5/GFP-encoding retrovirus as well. After flow cytometric sorting and gating of $\mathrm{GFP}^{+}$ blasts, no differences were found in plasma membrane expression of HLA-DR compared to wild-type blasts (Fig. 3c). The strong reduction in TAP1 (Fig. 3a) and HLA class I (Fig. 3c) expression showed that the function of TAP was indeed inhibited in Kasumi-1/UL49.5 blasts.

By different ways of TAP inhibition, we demonstrate that in contrast to $\mathrm{CLIP}^{+}$Kasumi-1 blasts, HLA-DR transport to the plasma membrane is dependent on the function of TAP in a subpopulation of KG-1 blasts. This suggests that Ii-independent HLA-DR expression in CLIP ${ }^{-}$ leukemic blasts results from loading with cytoplasmic peptides processed by HLA class I antigen-processing machinery.

Ii expression is inversely correlated to the TAP-dependency of HLA-DR expression and transport to the plasma membrane in $\mathrm{CLIP}^{-} \mathrm{KG}-1$ blasts

Although HLA-DR expression was totally absent in a subpopulation of TAP-inhibited KG-1 blasts, the other subpopulation was still able to transport HLA-DR molecules to the plasma membrane. To explain this difference in effect, we flow cytometrically sorted the DR ${ }^{-}$(TAPdependent) and $\mathrm{DR}^{+}$(TAP-independent) subpopulation of $\mathrm{GFP}^{+} \mathrm{KG}-1 / \mathrm{UL} 49.5$ blasts. Plasma membrane expression of HLA class I was equally reduced on both subpopulations compared to wild-type blasts, as determined by flow cytometry (Fig. 4a). This indicated that UL49.5 protein levels and the degree of TAP inhibition did not differ between these subpopulations.

We next studied whether the differences in TAP dependency of HLA-DR expression by KG-1 blasts involved the function of Ii. Flow cytometric analysis revealed a close association between total HLA-DR and Ii expression in $\mathrm{GFP}^{+} \mathrm{KG}-1 / \mathrm{BNLF} 2 \mathrm{a}$ and KG-1/UL49.5 blasts, as well as in the $\mathrm{DR}^{-}$- and $\mathrm{DR}^{+}$-sorted subpopulations of $\mathrm{GFP}^{+}$ KG-1/UL49.5 blasts (Fig. 4b). During cell culture, both HLA-DR and Ii expression were up-regulated again in the 
a

KG-1 MG-132

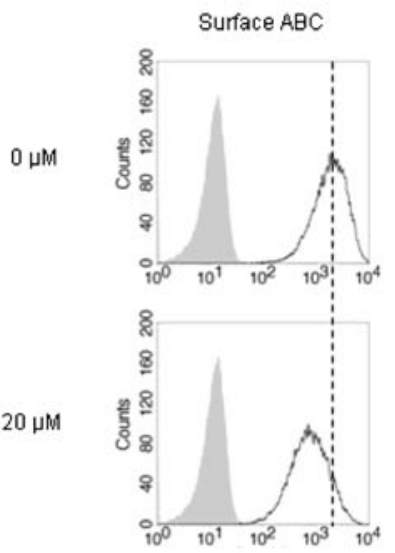

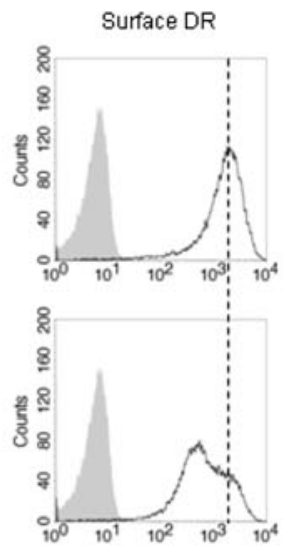

bortezomib

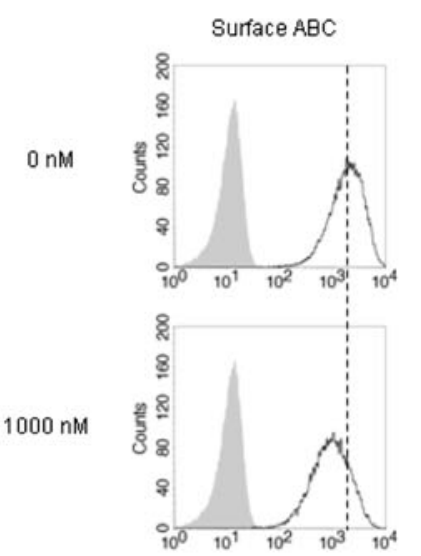

Log fluorescence

b

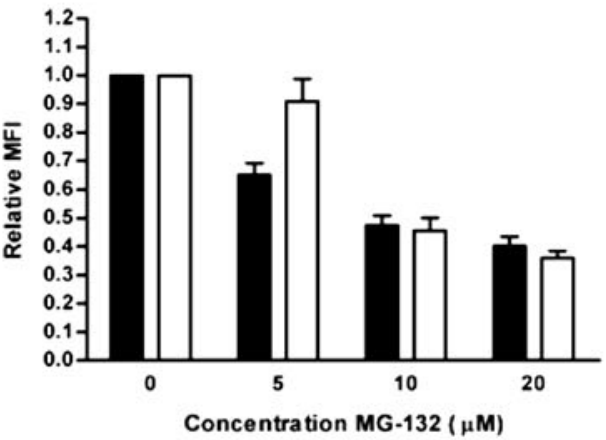

c

Kasumi-1

MG-132

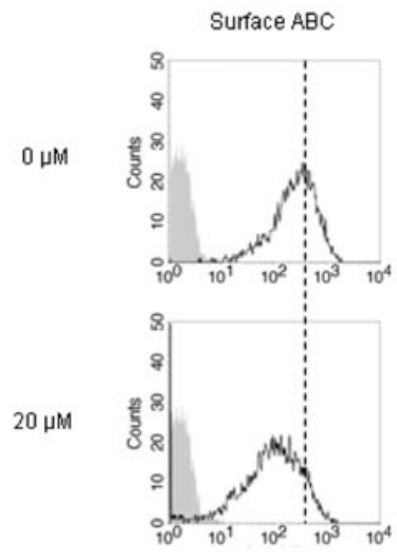

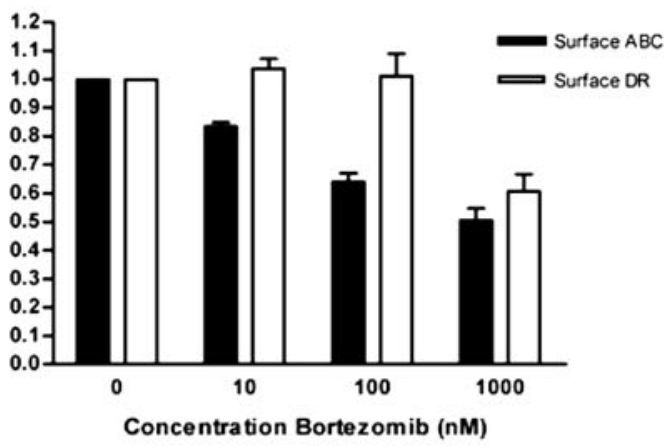

bortezomib
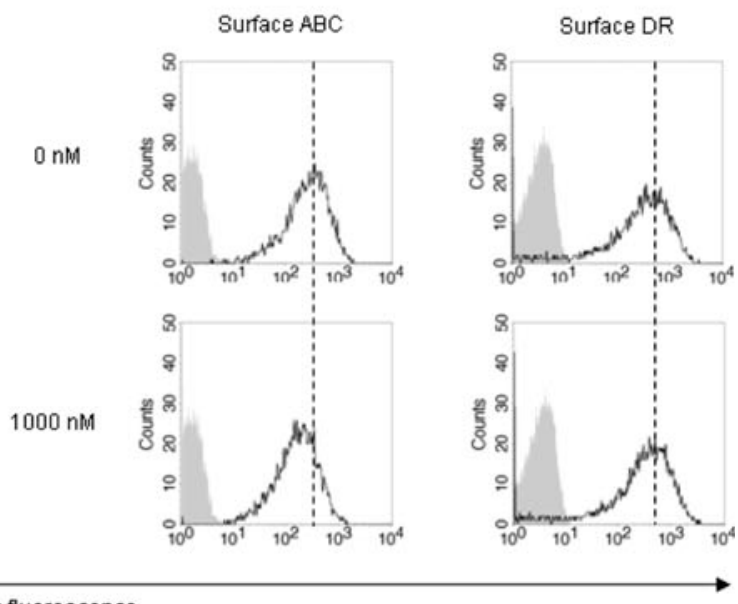
Fig. 3 TAP inhibition in KG-1 and Kasumi-1 blasts and the effect on HLA-DR expression. a KG-1 and Kasumi-1 blasts were transduced with retrovirus encoding GFP together with either BNLF2a or UL49.5 proteins, two different inhibitors of TAP. After flow cytometric sorting of $\mathrm{GFP}^{+}$blasts, TAP1 and TAP2 expression were determined by western blotting. All lanes were equally loaded with protein, as $\beta$-actin was expressed equally. Molecular weights: TAP1, $71 \mathrm{kD}$; TAP2,

$75 \mathrm{kD} ; \beta$-actin, $42 \mathrm{kD}$.

b, c Flow cytometrically sorted GFP $^{+}$KG-1 (b) and Kasumi-1 (c) blasts containing either UL49.5 or BNLF2a were analyzed for GFP as well as plasma membrane HLA class I ('ABC') and HLA-DR expression (unfilled histograms) by flow cytometry. GFP and plasma membrane HLA class I levels were evaluated to assess the transduction efficiency and the degree of TAP inhibition, respectively. The filled

histograms represent stainings of the same cells with control $\mathrm{IgG}$ a
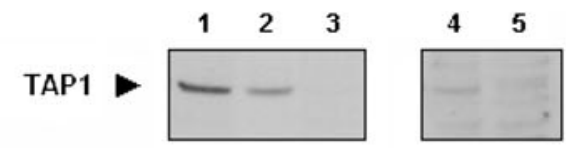

TAP2
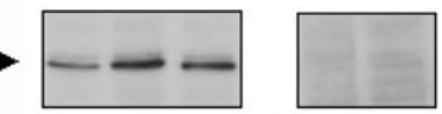

1 = KG-1

$2=K G-1 / B N L F 2 a$

$3=$ KG-1 UL 49.5

$\beta$-actin

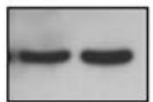

4 = Kasumi-1

5 = Kasumi-1 1 LL 49.5 b

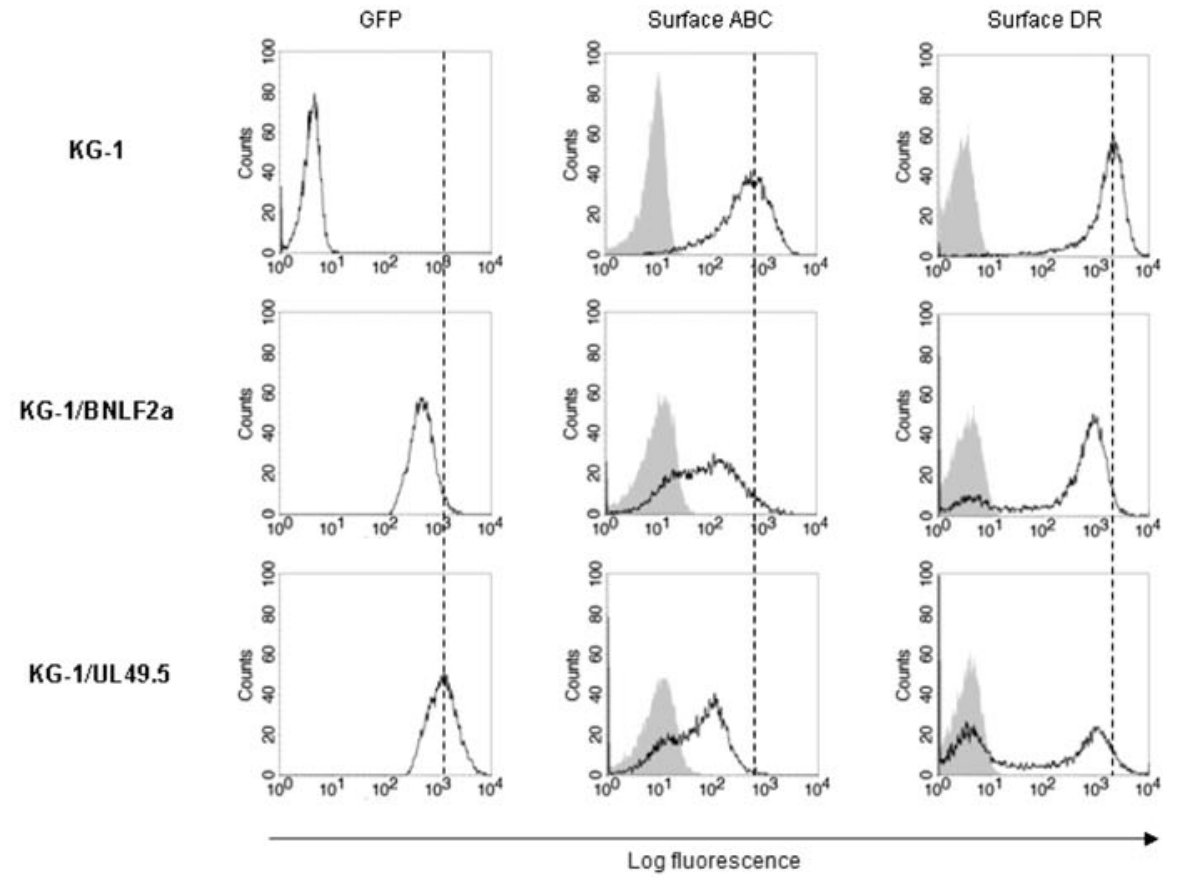

C

Kasumi-1

Kasumi-1/UL49.5

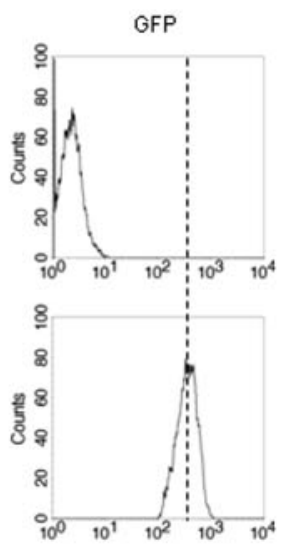

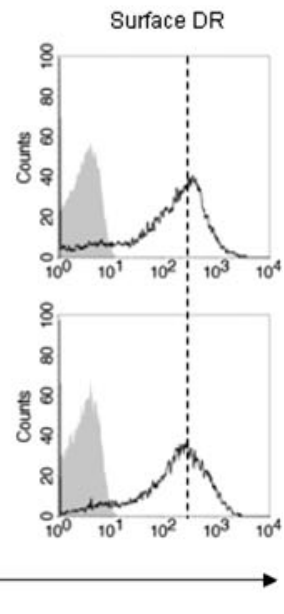


a

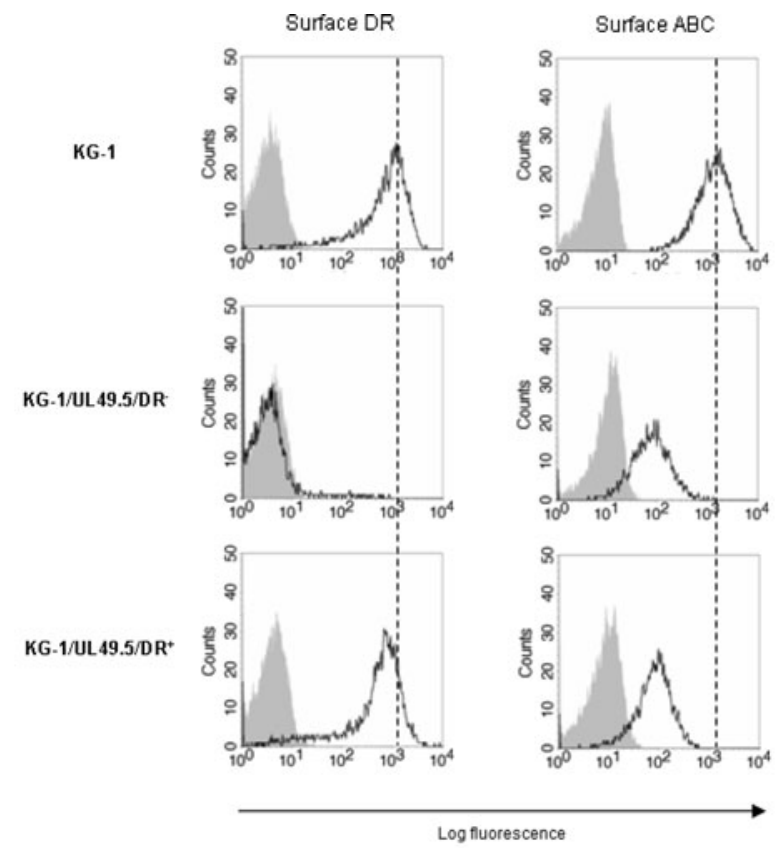

b

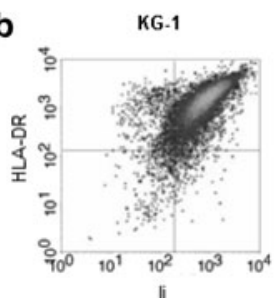

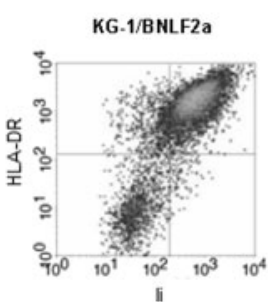

KG-1/UL.49.5

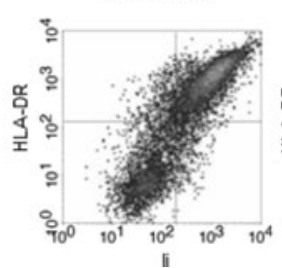

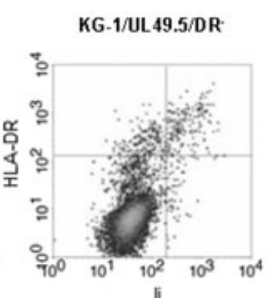

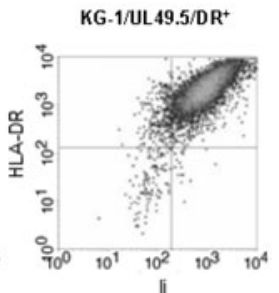

Fig. 4 a $\mathrm{DR}^{-}$and $\mathrm{DR}^{+}$subpopulations emerging from UL49.5containing KG-1 blasts were flow cytometrically sorted and $\mathrm{GFP}^{+}$ blasts were examined for plasma membrane levels of HLA-DR and HLA class I ('ABC') (unfilled histograms) with their isotope controls (filled histograms) by flow cytometry. Analysis of HLA-DR expression confirmed the purity of flow cytometric sorting. b Total HLA-DR

$\mathrm{DR}^{-}$-sorted subpopulation, while the $\mathrm{DR}^{+} \mathrm{Ii}^{+}$phenotype remained stable in the $\mathrm{DR}^{+}$-sorted subpopulation of $\mathrm{GFP}^{+}$ KG-1/UL49.5 blasts (data not shown).

Thus, in one KG-1 blast subpopulation, TAP suppression accompanied an unexpected, transient decline in Ii amount, probably resulting in the total absence of HLADR. In another subpopulation of KG-1 blasts, the expression of Ii was not affected by TAP inhibition, which indicated that HLA-DR expression in these blasts is rescued by association with Ii.

TAP and Ii together form the key mediators of HLA-DR expression and transport to the plasma membrane in CLIP $^{-}$KG-1 blasts

To actually show the capacity of Ii in rescuing HLA-DR expression in TAP-inhibited KG-1 blasts, we retrovirally transfected the $\mathrm{DR}^{+}$-sorted subpopulation of $\mathrm{GFP}^{+} \mathrm{KG}-1 /$ UL49.5 (KG-1/UL49.5/DR ${ }^{+}$) blasts with Ii-siRNAs. In expression was correlated to the expression of Ii in TAP-inhibited KG-1 variants. Flow cytometric analysis was performed on permeabilized, GFP ${ }^{+}$KG-1 blasts that contained either BNLF2a or UL49.5 proteins. For $\mathrm{GFP}^{+}$KG-1/UL49.5 blasts, Ii expression was analyzed in the flow cytometrically sorted $\mathrm{DR}^{-}$and $\mathrm{DR}^{+}$subpopulation as well

agreement with the flow cytometric data (Fig. 4b), total HLA-DR expression levels in $\mathrm{GFP}^{+} \mathrm{KG}-1 / \mathrm{UL} 49.5 / \mathrm{DR}^{+}$ blasts were slightly increased in comparison to those in wild-type blasts, as determined by western blotting (Fig. 5a). This indicates that in the absence of TAP, the association of HLA-DR molecules with Ii enhances their stability. The total amount of HLA-DR strongly declined after Ii silencing compared to non-silenced $\mathrm{GFP}^{+} \mathrm{KG}-1 /$ $\mathrm{UL} 49.5 / \mathrm{DR}^{+}$and wild-type blasts. We confirmed these results by flow cytometry (Fig. 5b; supplementary material, Fig. S4), showing similar reduction in HLA-DR expression after $>90 \%$ Ii down-regulation in $\mathrm{GFP}^{+}$KG-1/UL49.5/ $\mathrm{DR}^{+}$blasts. This was contrary to the observations in KG-1/ Ii-siRNA blasts with the TAP complex still functional (Fig. 1b).

Thus, TAP-independent HLA-DR expression and transport to the plasma membrane in KG-1 blasts is confined to the function of Ii, consistent with the classical pathway of HLA class II antigen presentation. 
Fig. 5 Invariant chain silencing in TAP-inhibited KG-1 blasts and the effect on HLA-DR expression. a The flow cytometrically sorted $\mathrm{DR}^{+}$ subpopulation of $\mathrm{GFP}^{+} \mathrm{KG}-1 /$ UL49.5 blasts was treated with Ii-siRNAs, and protein extracts were analyzed for the amount of TAP, Ii and HLA-DR by western blotting. The amount of $\beta$-tubulin in each lane confirmed equal protein loading.

Molecular weights: TAP1, 74

$\mathrm{kD}$; Ii, $33 \mathrm{kD}$ (p33); DR $\alpha, 34$

$\mathrm{kD} ; \beta$-tubulin, $55 \mathrm{kD}$. b Flow cytometric analyses of Ii versus total HLA-DR expression in permeabilized, $\mathrm{GFP}^{+} \mathrm{KG}-1 /$ $\mathrm{UL} 49.5 / \mathrm{DR}^{+}$blasts (left panel) and the resulting HLA-DR expression levels at the plasma membrane of intact, 7AAD blasts (right panel; unfilled histograms) after treatment with and without Ii-siRNAs. The filled histograms indicate the corresponding isotype control stainings a
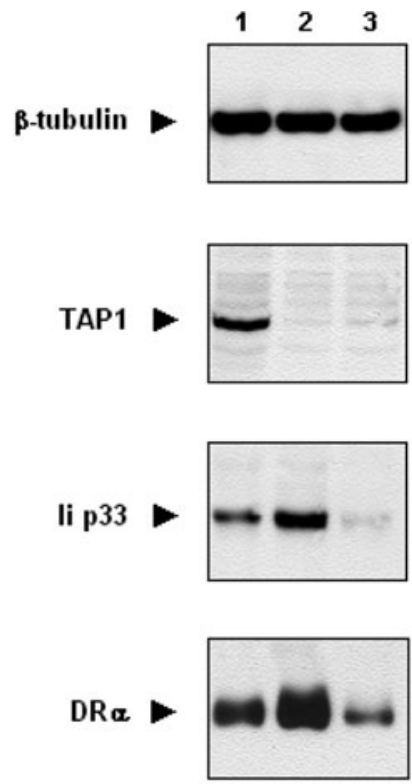

1 = KG-1

$2=K G-1 / U L 49.5 / D^{+}$

$3=\mathrm{KG}-1 / \mathrm{UL} 49.5 / \mathrm{DR}^{+} / \mathrm{li}-\mathrm{siRNA}$

b

KG-1/UL 49.5/DR ${ }^{+}$

KG-1/UL 49.5/DR ${ }^{+} / \mathrm{li}$-siRNA
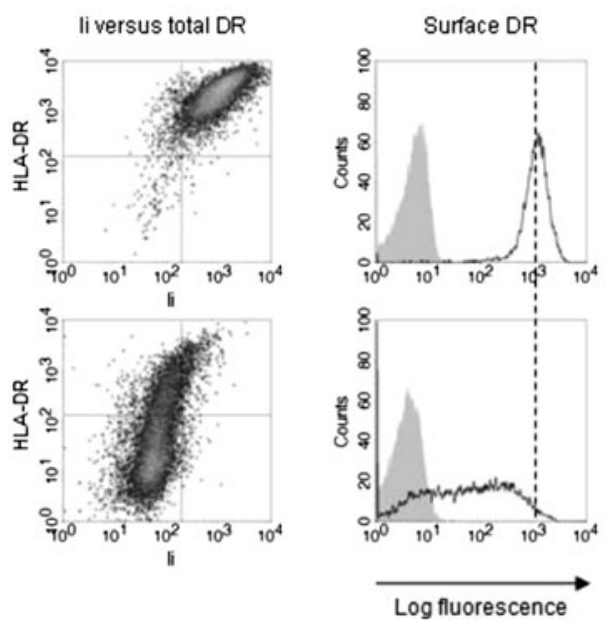

To study if the function of TAP is also the determining factor in Ii-independent HLA-DR expression, we transduced KG-1/Ii-siRNA blasts with BNLF2a/GFP or UL49.5/GFP retrovirus and flow cytometrically sorted $\mathrm{GFP}^{+}$blasts. The inhibition of TAP resulted in a strong down-regulation of total and plasma membrane expression of HLA-DR in both $\mathrm{GFP}^{+}$KG-1/Ii-siRNA/BNLF2a and KG-1/Ii-siRNA/UL49.5 blasts (Fig. 6). The difference in effect of TAP inhibition on HLA-DR expression could be explained by the higher transduction efficiency of UL49.5/ GFP compared to BNLF2a/GFP retrovirus (data not shown), as noticed earlier in wild-type blasts (Fig. 3b).

In accordance with the requirement of Ii in TAP-independent HLA-DR expression, the function of TAP is critical for Ii-independent HLA-DR expression and transport to the plasma membrane in KG-1 blasts. Overall, simultaneous inhibition of TAP- and Ii-dependent antigenprocessing pathways in these blasts strongly downmodulates HLA-DR expression, showing that TAP and Ii together are the key regulators of HLA class II antigen presentation.

\section{Discussion}

For an effective anti-tumor immune response, CTLs and $\mathrm{CD} 4^{+} \mathrm{T}$ cells need to be stimulated with TAAs presented by tumor APCs [34]. Professional APCs present these 
Fig. 6 The impact of TAP inhibition in Invariant Chainsilenced KG-1 blasts on

HLA-DR expression. Ii-silenced

KG-1 blasts transduced with

BNLF2a/GFP or UL49.5/GFP

retrovirus were flow

cytometrically sorted for $\mathrm{GFP}^{+}$

blasts. Subsequently, we

determined Ii versus total HLA-

DR expression in permeabilized

$\mathrm{GFP}^{+}$blasts (left panel) and the

resulting expression levels of

HLA-DR on intact, 7AAD

blasts (right panel; unfilled

histograms) by flow cytometry.

The filled histograms represent

stainings of the same cells with

control IgG
KG-1/i-siRNA

KG-1/i-siRNA/BNLF2a

KG-1/li-SiRNAUL49.5
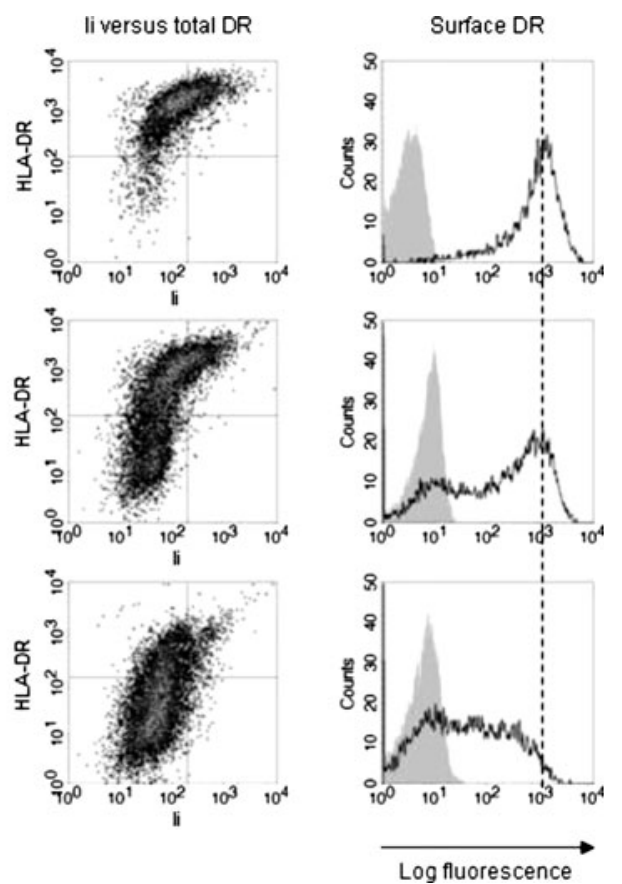

peptides after recognition, engulfment and processing of exogenously derived TAAs. Certain tumor cells, however, can also serve as potent APCs by not only expressing costimulatory molecules, but also presenting their own peptides to T cells. Since impaired TAA presentation is a known tumor escape mechanism [35], the components involved in processing and presentation of endogenous TAAs in tumor cells are interesting targets for immunomodulation. Previously, it has been reported that the CLIP ${ }^{-}$ KG-1 myeloid leukemic cell line is able to stimulate $\mathrm{T}$ cell reactivity and presents HLA-DR molecules that are loaded with peptides from endogenous rather than exogenous sources [36, 37]. In this report, we show that in addition to classical Ii-dependent HLA-DR antigen presentation, KG-1 blasts use HLA class I antigen-processing machinery, including the proteasome and TAP complex, to process endogenous antigens for presentation via HLA-DR molecules.

The Ii-independency of HLA-DR expression in KG-1 blasts (Fig. 1a, b) is not unique to this myeloid leukemic cell line, as we recently found the same in the THP-1 myeloid leukemic cell line treated with Ii-siRNAs [23]. Although Ii plays an essential role in targeting HLA-DR into the endosomal/lysosomal pathway [7], HLA-DR molecules may also reach the MIICs in an Ii-independent way, either indirectly via the plasma membrane or directly by using the di-leucine endosomal sorting motif that resides in the $\mathrm{DR} \beta$-chain $[38,39]$. In another myeloid leukemic cell line, the Kasumi-1, however, we showed that Ii silencing did affect plasma membrane expression of HLA-DR [23], indicating that HLA-DR molecules in some myeloid leukemic blasts cannot reach the MIICs without Ii. These differences in Ii- (as well as TAP-) dependency of HLA-DR expression might be explained by the low Ii/TAP ratio in KG-1 blasts (Figs. 1a, 3a) as compared to Kasumi-1 blasts (Fig. 3a, data not shown).

Since at least a proportion of HLA-DR molecules were immunoprecipitated along with Ii in KG-1 blasts (Fig. 1c), it seems that both an Ii-dependent and Ii-independent pathway were active. With regard to processing of endogenous antigens in these pathways, we showed that HLA-DR expression levels at the KG-1 plasma membrane were strongly influenced by proteasome inhibition (Fig. 2a, b). This implies that in KG-1 blasts, proteasomal instead of lysosomal degradation is involved in the generation of cytoplasmic peptides for loading onto HLA class II molecules. These findings differ from previous studies with murine tumor cells, in which HLA class II antigen presentation was dependent on the endosomal/lysosomal pathway rather than the proteasome [40, 41].

After proteasomal processing of cytoplasmic antigens in $\mathrm{KG}-1$ blasts, the proteasome-generated peptides might be transported to the MIICs for further trimming and subsequent loading onto HLA-DR molecules via the Ii-dependent pathway [12]. For HLA-DR molecules that are processed in the Ii-independent pathway, however, the intracellular site of peptide loading is still controversial. In this report, we demonstrate that for a particular subpopulation of KG-1 blasts, HLA-DR expression is dependent on the function of TAP (Fig. 3b). Since HLA-DR expression can occur in an Ii-independent way, it points to the ER as a potential compartment where HLA-DR molecules are 
loaded with proteasome-generated and TAP-translocated peptides.

The difference in TAP dependency of HLA-DR expression within the total KG-1 blast population might be explained by a differential expression of specific HLA-DR alleles with a high affinity for endogenous peptides. It was reported that two allelic subtypes, DRB $1 * 1101$ and DRB1*1401, on KG-1 blasts predominantly presented endogenous peptides [36]. In addition, HLA-DR4 and -DR7 molecules have been shown to associate with endogenous peptide presentation on tumor cells $[42,43]$. Unexpectedly, BNLF2a or UL49.5 proteins abrogated the expression of Ii in the DR ${ }^{-}$KG-1 blast population (Fig. $4 \mathrm{~b}$ ), but were recovered again during culture (data not shown). The suppression of TAP might therefore transiently elicit additional effects on the HLA class II-restricted presentation pathway in this subset of blasts, as we found reductions in HLA-DM expression levels as well (data not shown). This suggests that the master regulator of the HLA class II presentation pathway, CIITA, might be affected, which is currently under investigation.

By simultaneous inhibition of Ii and TAP, we were able to discriminate between the Ii-dependent and Ii-independent HLA class II presentation pathway of endogenous peptides in these tumor cells. We found that in the $\mathrm{DR}^{+}$ subpopulation of TAP-inhibited KG-1 blasts, only the Iidependent pathway was functional, as Ii silencing strongly reduced expression levels of HLA-DR (Fig. 5a). This seems to be a threshold effect as the amount of HLA-DR expressed is not directly proportional to the quantity of Ii (Fig. 5b). In KG-1/Ii-siRNA blasts, HLA-DR expression was clearly down-modulated after TAP suppression (Fig. 6), showing that the Ii-independent pathway depends on the function of TAP. The inability to express HLA-DR molecules in the absence of both Ii and TAP function agreed with the $\mathrm{DR}^{-}$phenotype found for the TAP-deficient KG-1 subpopulation lacking Ii expression (Fig. 4b).

This study provides important insights into the intracellular mechanisms of tumor cells to process endogenous antigens for HLA class II-restricted presentation. Extensive interplay between HLA class I and II presentation pathways was shown in blasts of the $\mathrm{CLIP}^{-} \mathrm{KG}-1$ myeloid leukemic cell line, which constitutively express HLA class II molecules loaded with endogenous peptides. These findings indicate that CLIP $^{-}$leukemic blasts may be able to present tumor-associated peptides on both HLA class I and II molecules and underlines the hypothesis that abnormalities in the HLA class I antigen-processing machinery in certain tumor cells may result in tumor escape from both HLA class I- and HLA class II-restricted T cell recognition. Besides their potential as antigen presenters, KG-1 blasts can also be differentiated into functional DC-like cells $[37,44]$, which makes these tumor cells ideal candidates for the use as cellular vaccines. In general, the involvement of endogenous antigen processing in HLA class II presentation by tumor cells might introduce new immunomodulatory approaches to simultaneously induce HLA class I and II tumor antigen presentation.

Acknowledgments This work was financially supported by Stichting Vanderes (grant 125), the Dutch Cancer Foundation (RUL 20053259 and NKI 2001-2415), the Netherlands Scientific Organization (Vidi 917.76.330) and the Landsteiner Foundation for Blood Research (grant 0533).

Conflict of interest The authors declare that they have no conflict of interest.

Open Access This article is distributed under the terms of the Creative Commons Attribution Noncommercial License which permits any noncommercial use, distribution, and reproduction in any medium, provided the original author(s) and source are credited.

\section{References}

1. Nandi D, Tahiliani P, Kumar A, Chandu D (2006) The ubiquitinproteasome system. J Biosci 31:137-155

2. Androlewicz MJ, Anderson KS, Cresswell P (1993) Evidence that transporters associated with antigen processing translocate a major histocompatibility complex class I-binding peptide into the endoplasmic reticulum in an ATP-dependent manner. Proc Natl Acad Sci USA 90:9130-9134

3. Serwold T, Gonzalez F, Kim J, Jacob R, Shastri N (2002) ERAAP customizes peptides for MHC class I molecules in the endoplasmic reticulum. Nature 419:480-483

4. Lotteau V, Teyton L, Peleraux A, Nilsson T, Karlsson L, Schmid SL, Quaranta V, Peterson PA (1990) Intracellular transport of class II MHC molecules directed by invariant chain. Nature 348:600-605

5. Neefjes JJ, Stollorz V, Peters PJ, Geuze HJ, Ploegh HL (1990) The biosynthetic pathway of MHC class II but not class I molecules intersects the endocytic route. Cell 61:171-183

6. Anderson MS, Miller J (1992) Invariant chain can function as a chaperone protein for class II major histocompatibility complex molecules. Proc Natl Acad Sci USA 89:2282-2286

7. Pieters J, Bakke O, Dobberstein B (1993) The MHC class IIassociated invariant chain contains two endosomal targeting signals within its cytoplasmic tail. J Cell Sci 106(Pt 3):831-846

8. Romagnoli P, Germain RN (1994) The CLIP region of invariant chain plays a critical role in regulating major histocompatibility complex class II folding, transport, and peptide occupancy. J Exp Med 180:1107-1113

9. Sloan VS, Cameron P, Porter G, Gammon M, Amaya M, Mellins E, Zaller DM (1995) Mediation by HLA-DM of dissociation of peptides from HLA-DR. Nature 375:802-806

10. Guermonprez P, Saveanu L, Kleijmeer M, Davoust J, van EP, Amigorena S (2003) ER-phagosome fusion defines an MHC class I cross-presentation compartment in dendritic cells. Nature 425:397-402

11. Burgdorf S, Scholz C, Kautz A, Tampe R, Kurts C (2008) Spatial and mechanistic separation of cross-presentation and endogenous antigen presentation. Nat Immunol 9:558-566

12. Dani A, Chaudhry A, Mukherjee P, Rajagopal D, Bhatia S, George A, Bal V, Rath S, Mayor S (2004) The pathway for 
MHCII-mediated presentation of endogenous proteins involves peptide transport to the endo-lysosomal compartment. J Cell Sci 117:4219-4230

13. Dengjel J, Schoor O, Fischer R, Reich M, Kraus M, Muller M, Kreymborg K, Altenberend F, Brandenburg J, Kalbacher H, Brock R, Driessen C, Rammensee HG, Stevanovic S (2005) Autophagy promotes MHC class II presentation of peptides from intracellular source proteins. Proc Natl Acad Sci USA 102:79227927

14. Paludan C, Schmid D, Landthaler M, Vockerodt M, Kube D, Tuschl T, Munz C (2005) Endogenous MHC class II processing of a viral nuclear antigen after autophagy. Science 307:593-596

15. Zhou D, Li P, Lin Y, Lott JM, Hislop AD, Canaday DH, Brutkiewicz RR, Blum JS (2005) Lamp-2a facilitates MHC class II presentation of cytoplasmic antigens. Immunity 22:571-581

16. Lich JD, Elliott JF, Blum JS (2000) Cytoplasmic processing is a prerequisite for presentation of an endogenous antigen by major histocompatibility complex class II proteins. J Exp Med 191:1513-1524

17. Mukherjee P, Dani A, Bhatia S, Singh N, Rudensky AY, George A, Bal V, Mayor S, Rath S (2001) Efficient presentation of both cytosolic and endogenous transmembrane protein antigens on MHC class II is dependent on cytoplasmic proteolysis. J Immunol 167:2632-2641

18. Armstrong TD, Clements VK, Martin BK, Ting JP, OstrandRosenberg S (1997) Major histocompatibility complex class IItransfected tumor cells present endogenous antigen and are potent inducers of tumor-specific immunity. Proc Natl Acad Sci USA 94:6886-6891

19. Zhao Y, Boczkowski D, Nair SK, Gilboa E (2003) Inhibition of invariant chain expression in dendritic cells presenting endogenous antigens stimulates CD4+ T-cell responses and tumor immunity. Blood 102:4137-4142

20. Long EO, LaVaute T, Pinet V, Jaraquemada D (1994) Invariant chain prevents the HLA-DR-restricted presentation of a cytosolic peptide. J Immunol 153:1487-1494

21. Roche PA, Cresswell P (1990) Invariant chain association with HLA-DR molecules inhibits immunogenic peptide binding. Nature 345:615-618

22. Tewari MK, Sinnathamby G, Rajagopal D, Eisenlohr LC (2005) A cytosolic pathway for MHC class II-restricted antigen processing that is proteasome and TAP dependent. Nat Immunol 6:287-294

23. van Luijn MM, Chamuleau ME, Thompson JA, Ostrand-Rosenberg S, Westers TM, Souwer Y, Ossenkoppele GJ, van Ham SM, van de Loosdrecht AA (2009) CLIP down-modulation enhances the immunogenicity of myeloid leukemic blasts resulting in increased CD4+ T cell responses. Haematologica 95:485-493

24. Roche PA, Marks MS, Cresswell P (1991) Formation of a ninesubunit complex by HLA class II glycoproteins and the invariant chain. Nature 354:392-394

25. Hislop AD, Ressing ME, van Leeuwen D, Pudney VA, Horst D, Koppers-Lalic D, Croft NP, Neefjes JJ, Rickinson AB, Wiertz EJ (2007) A CD8+ T cell immune evasion protein specific to Epstein-Barr virus and its close relatives in Old World primates. J Exp Med 204:1863-1873

26. Koppers-Lalic D, Reits EA, Ressing ME, Lipinska AD, Abele R, Koch J, Marcondes RM, Admiraal P, van LD, BienkowskaSzewczyk K, Mettenleiter TC, Rijsewijk FA, Tampe R, Neefjes J, Wiertz EJ (2005) Varicelloviruses avoid T cell recognition by UL49.5-mediated inactivation of the transporter associated with antigen processing. Proc Natl Acad Sci USA 102:5144-5149

27. Thompson JA, Dissanayake SK, Ksander BR, Knutson KL, Disis ML, Ostrand-Rosenberg S (2006) Tumor cells transduced with the MHC class II Transactivator and CD80 activate tumor-specific CD4+ T cells whether or not they are silenced for invariant chain. Cancer Res 66:1147-1154

28. Verweij MC, Koppers-Lalic D, Loch S, Klauschies F, de la SH, Quinten E, Lehner PJ, Mulder A, Knittler MR, Tampe R, Koch J, Ressing ME, Wiertz EJ (2008) The varicellovirus UL49.5 protein blocks the transporter associated with antigen processing (TAP) by inhibiting essential conformational transitions in the $6+6$ transmembrane TAP core complex. J Immunol 181:4894-4907

29. Adams TE, Bodmer JG, Bodmer WF (1983) Production and characterization of monoclonal antibodies recognizing the alphachain subunits of human ia alloantigens. Immunology 50:613624

30. Plewnia G, Schulze K, Hunte C, Tampe R, Koch J (2007) Modulation of the antigenic peptide transporter TAP by recombinant antibodies binding to the last five residues of TAP1. J Mol Biol 369:95-107

31. Schaiff WT, Hruska KA Jr, McCourt DW, Green M, Schwartz BD (1992) HLA-DR associates with specific stress proteins and is retained in the endoplasmic reticulum in invariant chain negative cells. J Exp Med 176:657-666

32. Viville S, Neefjes J, Lotteau V, Dierich A, Lemeur M, Ploegh H, Benoist C, Mathis D (1993) Mice lacking the MHC class IIassociated invariant chain. Cell 72:635-648

33. Berkers CR, Verdoes M, Lichtman E, Fiebiger E, Kessler BM, Anderson KC, Ploegh HL, Ovaa H, Galardy PJ (2005) Activity probe for in vivo profiling of the specificity of proteasome inhibitor bortezomib. Nat Methods 2:357-362

34. Knutson KL, Disis ML (2005) Tumor antigen-specific T helper cells in cancer immunity and immunotherapy. Cancer Immunol Immunother 54:721-728

35. van Luijn MM, van den Ancker W, Chamuleau ME, Ossenkoppele GJ, van Ham SM, van de Loosdrecht AA (2010) Impaired antigen presentation in neoplasia: basic mechanisms and implications for acute myeloid leukemia. Immunotherapy 2:85-97

36. Harris PE, Maffei A, Colovai AI, Kinne J, Tugulea S, Suciu-Foca N (1996) Predominant HLA-class II bound self-peptides of a hematopoietic progenitor cell line are derived from intracellular proteins. Blood 87:5104-5112

37. Teobald I, Dunnion DJ, Whitbread M, Curnow SJ, Browning MJ (2008) Phenotypic and functional differentiation of KG-1 into dendritic-like cells. Immunobiology 213:75-86

38. Smiley ST, Rudensky AY, Glimcher LH, Grusby MJ (1996) Truncation of the class II beta-chain cytoplasmic domain influences the level of class II/invariant chain-derived peptide complexes. Proc Natl Acad Sci USA 93:241-244

39. Simonsen A, Stang E, Bremnes B, Roe M, Prydz K, Bakke O (1997) Sorting of MHC class II molecules and the associated invariant chain (Ii) in polarized MDCK cells. J Cell Sci 110(Pt 5):597-609

40. Dissanayake SK, Tuera N, Ostrand-Rosenberg S (2005) Presentation of endogenously synthesized MHC class II-restricted epitopes by MHC class II cancer vaccines is independent of transporter associated with $\mathrm{Ag}$ processing and the proteasome. J Immunol 174:1811-1819

41. Qi L, Ostrand-Rosenberg S (2000) MHC class II presentation of endogenous tumor antigen by cellular vaccines depends on the endocytic pathway but not H2-M. Traffic 1:152-160

42. Posthuma EF, Falkenburg JH, Apperley JF, Gratwohl A, Hertenstein B, Schipper RF, Oudshoorn M, Biezen JH, Hermans J, Willemze R, Roosnek E, Niederwieser D (2000) HLA-DR4 is associated with a diminished risk of the development of chronic myeloid leukemia (CML). Chronic Leukemia Working Party of the European Blood and Marrow Transplant Registry. Leukemia $14: 859-862$ 
43. Thompson JA, Srivastava MK, Bosch JJ, Clements VK, Ksander BR, Ostrand-Rosenberg S (2008) The absence of invariant chain in MHC II cancer vaccines enhances the activation of tumor-reactive type $1 \mathrm{CD} 4(+) \mathrm{T}$ lymphocytes. Cancer Immunol Immunother 57:389-398
44. Hulette BC, Rowden G, Ryan CA, Lawson CM, Dawes SM, Ridder GM, Gerberick GF (2001) Cytokine induction of a human acute myelogenous leukemia cell line (KG-1) to a CD1a+ dendritic cell phenotype. Arch Dermatol Res 293:147-158 